\title{
Mini-invasive robotic assisted pyelolithotomy: Comparison between the transperitoneal and retroperitoneal approach
}

\author{
Daniele D'Agostino $^{1}$, Paolo Corsi ${ }^{1}$, Marco Giampaoli ${ }^{1}$, Federico Mineo Bianchi ${ }^{2}$, Daniele Romagnoli ${ }^{1}$, \\ Simone Crivellaro ${ }^{3}$, Giacomo Saraceni ${ }^{2}$, Marco Garofalo ${ }^{2}$, Riccardo Schiavina ${ }^{2}$, Eugenio Brunocilla ${ }^{2}$, \\ Walter Artibani ${ }^{1}$, Angelo Porreca ${ }^{1}$ \\ ${ }^{1}$ Department of Robotic Urological Surgery, Abano Terme Hospital, Abano Terme, Italy; \\ ${ }^{2}$ Department of Urology, University of Bologna, Bologna, Italy; \\ ${ }^{3}$ Division of Urology, Department of Surgery, University of Illinois at Chicago, Chicago, IL, USA
}

\begin{abstract}
Summary Objective: To compare the retroperitoneal with the transperitoneal approach in a series of patients underwent to robotic-assisted pyelolithotomy (RP). Materials and methods: From January 2015 to December 2018 we evaluated 20 patients subjected to robotic pyelolithotomy; 11 patients were treated with retroperitoneal approach (RRP) and 9 with transperitoneal approach (TRP). For each patient intra and perioperative data were recorded: operative time $(\mathrm{OT})$, blood loss (BL), length of hospital stay (LOS), stone clearance, post-operative complications and time to remove the drain. The presence of stone fragments $<4 \mathrm{~mm}$ was considered as stone free rate.

Results: The principal stone burden was greater in the TRP group than in the RRP group $(48 \pm 10 \mathrm{~mm}$ vs $32 \pm 14 \mathrm{~mm}, p=$ $0.12)$. Preoperative hydronephrosis was present in 7 (64\%) patients in RRP group and a mild hydronephrosis in 3 of TRP group $(p=0.04)$. The average operative time was higher in the $R R P$ group than in the TRP group $(203 \pm 45 \min$ vs $137 \pm 31$ $\min , p=0.002)$. The average blood loss was $305 \pm 175 \mathrm{ml}$ in the RRP group versus $94 \pm 104 \mathrm{ml}$ in the TRP group ( $p=$ $0.005)$. The stone free rate was similar between the two groups, $36 \%$ (4 patients) in the RRP group and $44 \%$ (4 patients) in the TRP $(p=0.966)$.

Conclusions: RP appears to be a safe and effective minimally invasive treatment for some patients with renal staghorn calculi or urinary tract malformations. The TRP may give lower operative time and better results in terms of blood loss and length of hospital stay.
\end{abstract}

KEY WORDS: Transperitoneal pyelolithotomy; Retroperitoneal pyelolithotomy.

Submitted 28 February 2019; Accepted 21 March 2019

\begin{abstract}
INTRODUCTION
Stone disease is a highly prevalent condition that unites all countries around the world; the incidence of urolithiasis depends on geographical, racial, and socioeconomic factors. There are a lot of variety of therapeutic option for renal stones: extracorporeal shock wave lithotripsy (ESWL), ureteroscopy (URS) and percutaneous nephrolithotomy (PNL) (1). Surgical management will depend on many factors including availability of different technologies. "Staghorn" stones are large branching stones that fill part of all of the renal pelvis and renal calyces and they can be complete or partial depending on the level of occupancy of the collect-
\end{abstract}

ing system (2). Although, the term 'staghorn' provides description of stone configuration, it lacks specific volume criteria and information about stone composition (3). PCNL remains the gold-standard for the management of larger and "staghorn" renal stones. Robot assisted laparoscopic surgery (RALS) may be useful in the management of upper tract (UT) urolithiasis and it has been frequently considered as an alternative procedure in the management of large or complex renal stones to PNL or open surgery. The mayor advantages are: the possibility to remove the stones integrally, the ability to minimize the bleeding, less pain and lower morbidity. The use of robotic surgery has allowed more success rates than reconstructive urinary tract surgery (4). In addition to remove the stones, the advantages of robotic pyelolithotomy are the possibility to perform reconstructive surgery in presence of anatomical anomalies (e.g. pelvic or horseshoe kidney and malrotated kidneys, failed PNL and stones associated with congenital renal anomalies such as ureteropelvic junction obstruction (UPJO) (3). As for the robotic surgery of renal neoplasms (5) RP can be performed transperitoneally or retroperitoneally and there is no evidence of which approach is better than the other. Aim of the study is to compare the perioperative outcomes and stone free status between the transperitoneal and retroperitoneal approach of robotic pyelolithotomy

\section{MATERIAL AND METHOdS}

From January 2015 to December 2018 we retrospectively evaluated 20 patients subjected to robotic pyelolithotomy from 3 high volume centers. The indication for robotic surgical treatment of urolithiasis was discussed with all patients when the informed consent was signed, indicating that the percutaneous nephrolithotomy was the gold standard treatment; however the main indications for the robotic surgical treatment were highlighted as extrarenal pelvis, anatomical abnormalities (e.g. pelvic kidney or horseshoe kidney), simultaneous management of coexisting pathologies such pelvic-ureteral junction obstruction or failed endourological procedure. All the patients enrolled presented an extrarenal pelvis to the preoperative CT scan and pyelolitotomy was proposed as

No conflict of interest declared. 
a treatment for stones even in the absence of reconstructive surgery.

The population is divided into 2 groups: 11 patients were treated with retroperitoneal approach (RRP) and 9 with transperitoneal approach (TRP).

Patients with coagulation disorder, cardiorespiratory diseases, musculoskeletal deformities and morbid obesity were excluded from the study.

For each patient the preoperative evaluation included renal function tests, urine routine and microscopic examination. The patients with urinary infection received a course of antimicrobial therapy and they underwent the procedure after the urine culture was sterile. A CT-scan (Computed Tomography) was performed for each patient to obtain information about the stone location, hydronephrosis, the status of contralateral kidney and the presence of anatomical abnormalities such as UPJO or malrotated kidney.

All patients were operated under general anesthesia; in the presence of concomitant UPJO, pyeloplasty was performed according to the Anderson-Hynes technique. At the end of each procedure a ureteral stent (double J) and an abdominal (intraperitoneal or retroperitoneal) drain were inserted. The double J stent was removed after 2-3 weeks and the abdominal drain was removed when no traces of creatinine were found in it.

For each patient intra and perioperative data were recorded: operative time (OT), blood loss (BL), length of hospital stay (LOS), stone clearance, post-operative complications and time to remove the drain. The presence of stone fragments $<4 \mathrm{~mm}$ was considered as stone free rate.

\section{Surgical technique}

Transperitoneal approach: The patient was placed in lateral decubitus position at $45^{\circ}$ angle.

A $12-\mathrm{mm}$ camera port was placed lateral and superior to the umbilicus and three $8-\mathrm{mm}$ robotic working ports were placed under direct vision in the ipsilateral upper quadrant, lower quadrant, and lateral abdomen. A 12-mm assistant port is usually placed close to the midline, midway between the camera port and the robotic ports (Figure 1). Sometimes a fourth robotic arm was used to aid retraction of the kidney and exposure of the renal pelvis and hilum.

\section{Figure 1.}

Position of trocars on transperitoneal approach.

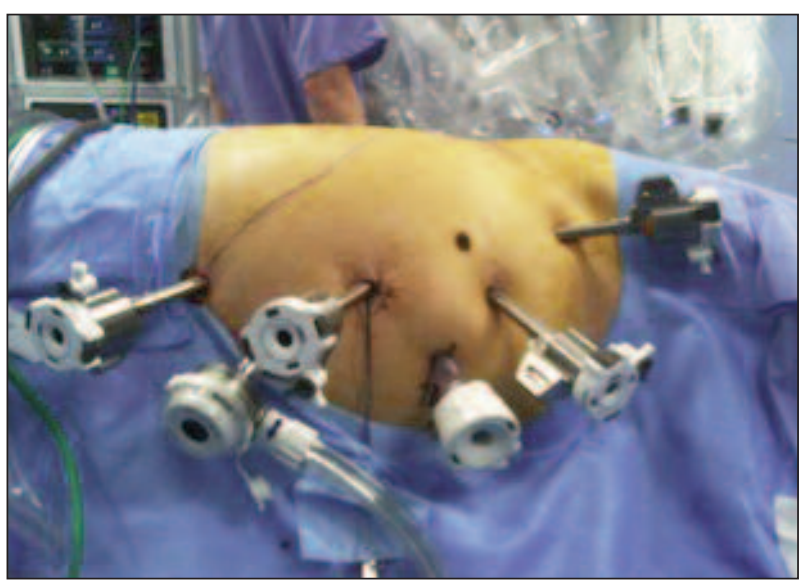

The line of Toldt was incised, the renocolic ligament was incised, and the colon was dissected and reflected medially to provide clear exposure of the ureteropelvic junction. The ureter was identified, recognizing its peristalsis and dissected. The renal pelvis was exposed by releasing adjacent structures with sharp and blunt dissection.

A vertical incision was made in the pelvis, until the stone is exposed. A robotic ProGrasp forcep was then used to carefully dislodge and remove the stone/s; if the stone was too large it was placed in a specimen retrieval bag and removed at the end of the procedure. Direct calyceal vision inspection was performed initially with the $30^{\circ}$ optic, and the flexible cystoscope was placed through the $10-\mathrm{mm}$ port, if required, to remove possible remaining stones. A ureteral double-J stent was placed, and thepyelotomy was closed in running fashion with absorbable suture. The perirenal fat was then approximated over the renal pelvis. A drain was placed through one of the trocar sites, the fascia and were closed in standard fashion.

Retroperitonal approach: The patient was positioned on full flank position, the umbilicus on the break point of the of the table, the legs were positioned and a pillow was put in between (the internal leg was flexed at $45^{\circ}$, while the external leg was totally extended) (Figure 2). The table was broken until maximum skin extension was reached in order to have as much working space as possible. The technique of applying trocars has been described (6): an oblique $1.5 \mathrm{~cm}$ incision was made at the tip of the $12^{\text {th }}$ rib following the direction of the exter-

Figure 2.

Position of patient on retroperitoneal approach.

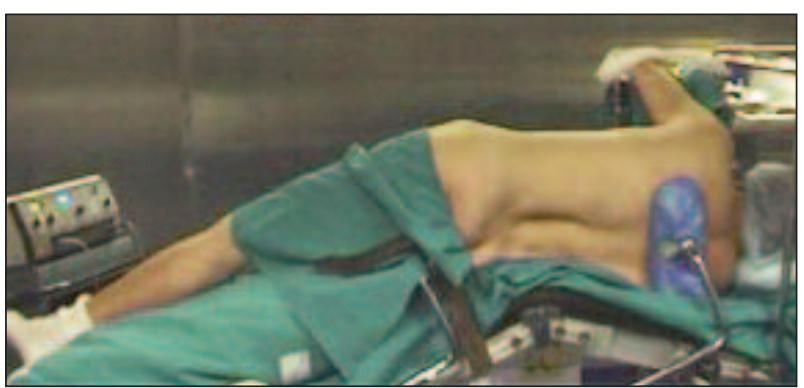

Figure 3.

Position of trocars on retroperitoneal approach.

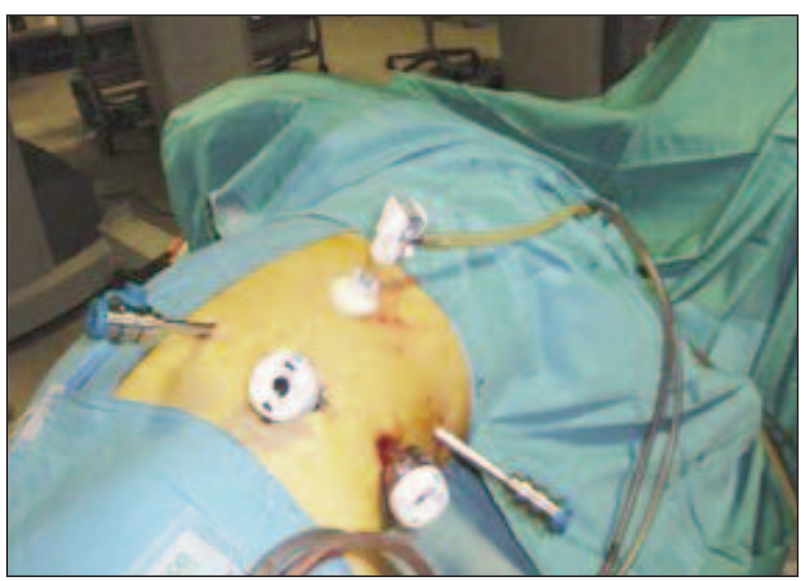


nal oblique muscle. The muscle fibers were gently dissected without cutting to the internal oblique muscle fibers, the dissection was then extended through the fascia. A minimal incision (2-3 mm) was done on the internal oblique fascia and the space was first blindly created with the finger through trasversalis fascia then with the introduction and subsequent inflation of a glove connected to the end of a nasal-gastric probe. A $12 \mathrm{~mm}$ trocar for the camera was positioned under finger guidance on the iliac crest along the mid-axillary line. An $8 \mathrm{~mm}$ robotic trocar was then positioned along the psoas muscle below the $12^{\text {th }}$ rib-vertebra angle. A $12 \mathrm{~mm}$ laparoscopic trocar was positioned along the psoas muscle behind the iliac crest. Then $12 \mathrm{~mm}$ Hasson trocar was finally positioned and the working space was created by $\mathrm{CO}_{2}$ inflation. The second $8 \mathrm{~mm}$ robotic trocar was positioned, after the laparoscopic dissection of the anterior peritoneal reflection, on the anterior axillary line on the same axis as the umbilicus (Figure 3). Using the robotic scissors and grasper the paranephric fat was first dissected then removed by a ring-clip to increase the workspace. After the identification of ureter, the kidney with its fat was isolated first posteriorly along psoas muscle, and, when necessary, anteriorly.

The Gerota fascia was incised exposing the perinephric fat. The hilum was then identified, renal pelvis was incised longitudinally and the stones should be easily dislodge and remove using the robotic ProGrasp. If needed, a flexible cystoscope was introduced through the assistant port to inspect the remaining of the renal pelvis and the calices. A stone basket was used to remove small residual stones from within the kidney. As in other robotic procedures the renal pelvis was then closed using a barbed bidirectional 3.0 suture. The choice of the suture depends by the thickness of the incision of renal pelvis.

In the presence of UPJO, pyeloplasty was performed both in the transperitoneal approach and in the retroperitoneal approach.

\section{Statistical analysis}

All data were statistically analyzed using SPSS v 21 for Macintosh. Continuous variables were expressed as means \pm standard deviation (SD) whereas categorical variables were expressed as frequencies with percentages. The indipendent-samples T-test and Chi-Square test were used to compare means and frequencies between the two groups, respectively.

\section{RESULTS}

The preoperative characteristics of the 2 groups (Table 1) were comparable about age, sex, body mass index (BMI) and number and localization of the stones. The principal stone burden was greater in the TRP group (transperitoneal approach) than in the RRP group (retroperitoneal approach) $(48 \pm 10 \mathrm{~mm}$ vs $32 \pm 14 \mathrm{~mm}, \mathrm{p}=0.12)$. Preoperative hydronephrosis was present in 7 (64\%) patients in RRP group; in three patients in TRP group was present a grade I hydronephrosis $(p=0.04)$; a ureteral stent was placed preoperatively in $1(9 \%)$ patients in RRP group and in $5(56 \%)$ of patients in TRP group ( $\mathrm{p}=0.024)$. Table 2 presents a comparison between the intra and peri- operative outcomes between the two groups. The average operative time was higher in the RRP group than in the TRP group ( $203 \pm 45$ min vs $137 \pm 31 \mathrm{~min}, \mathrm{p}=0.002$ ). The average blood loss was $305 \pm 175 \mathrm{ml}$ in the RRP group versus $94 \pm 104 \mathrm{ml}$ in the TRP group $(\mathrm{p}=0.005)$. The stone free rate was similar between the two groups, $36 \%$ (4 patients) in the RRP group and 44\% (4 patients)

\section{Table 1.}

Patients characteristics.

\begin{tabular}{|c|c|c|c|}
\hline & $\begin{array}{l}\text { Retroperitoneal } \\
\text { approach }\end{array}$ & $\begin{array}{l}\text { Transperitoneal } \\
\text { approach }\end{array}$ & $p$ value \\
\hline \multicolumn{4}{|l|}{ Age } \\
\hline Mean \pm SD & $56 \pm 10$ & $51 \pm 11$ & 0.352 \\
\hline \multicolumn{4}{|l|}{ Gender (\%) } \\
\hline Male & $3(27)$ & $4(44)$ & 0.33 \\
\hline Female & $8(73)$ & $5(56)$ & \\
\hline \multicolumn{4}{|l|}{ BMI } \\
\hline Mean \pm SD & $26 \pm 4.5$ & $26 \pm 3$ & 0.788 \\
\hline \multicolumn{4}{|c|}{ Stone Number (\%) } \\
\hline 1 & $5(46)$ & $6(67)$ & 0.26 \\
\hline 2 & $2(18)$ & $2(22)$ & \\
\hline 3 & $4(36)$ & $0(0)$ & \\
\hline 4 & $0(0)$ & $0(0)$ & \\
\hline 5 & $0(0)$ & $1(1)$ & \\
\hline \multicolumn{4}{|c|}{ Principal Stone Location (\%) } \\
\hline Pelvis & $7(64)$ & $3(33)$ & 0.25 \\
\hline Staghorn & $4(36)$ & $6(67)$ & \\
\hline Lower Pole & $0(0)$ & $0(0)$ & \\
\hline \multicolumn{4}{|c|}{ Principal Stone Burden (mm) } \\
\hline Mean \pm SD & $32 \pm 14$ & $48 \pm 10$ & 0.12 \\
\hline \multicolumn{4}{|l|}{ Side (\%) } \\
\hline Left & $5(45)$ & $5(56)$ & 0.65 \\
\hline Right & $6(55)$ & $4(44)$ & \\
\hline \multicolumn{4}{|c|}{ Hidronephrosis (\%) } \\
\hline Yes & $7(64)$ & $3(30)$ & 0.003 \\
\hline No & $4(36)$ & $7(70)$ & \\
\hline \multicolumn{4}{|c|}{$\overline{\text { Previous Stent (\%) }}$} \\
\hline Yes & $1(9)$ & $5(56)$ & 0.024 \\
\hline No & $10(91)$ & $4(44)$ & \\
\hline
\end{tabular}

Table 2.

Comparison between TRP and RRP.

\begin{tabular}{|lccc|}
\hline & $\begin{array}{c}\text { Retroperitoneal } \\
\text { approach (n 11) }\end{array}$ & $\begin{array}{c}\text { Transperitoneal } \\
\text { approach (n 9) }\end{array}$ & p value \\
\hline $\begin{array}{l}\text { Operation time (min) } \\
\text { Mean } \pm \text { SD }\end{array}$ & $203 \pm 45$ & $137 \pm 31$ & 0.002 \\
\hline $\begin{array}{l}\text { Blood loss (ml) } \\
\text { Mean } \pm \text { SD }\end{array}$ & $305 \pm 175$ & $94 \pm 104$ & 0.005 \\
\hline $\begin{array}{l}\text { Stone free rate (\%) } \\
\text { Yes }\end{array}$ & $4(36)$ & $4(44)$ & 0.966 \\
No & $7(64)$ & $5(56)$ & \\
\hline Residual stone burden (mm) & $61 \pm 19$ & $58 \pm 12$ & 0.08 \\
\hline $\begin{array}{l}\text { Concomitant pieloplasty } \\
\text { sec. Anderson-Hynes (\%) }\end{array}$ & $1(9)$ & $3(33)$ & 0.18 \\
$\begin{array}{l}\text { Yes } \\
\text { No }\end{array}$ & $10(91)$ & $6(67)$ & \\
\hline $\begin{array}{l}\text { Drain (days) } \\
\text { Mean } \pm \text { SD }\end{array}$ & $4.27 \pm 2.7$ & $1 \pm 2.7$ & 0.013 \\
\hline $\begin{array}{l}\text { Lenght of hospital stay (days) } \\
\text { Mean } \pm \text { SD }\end{array}$ & $5.3 \pm 2.7$ & $2 \pm 2.7$ & 0.013 \\
\hline $\begin{array}{l}\text { Follow-up (months) } \\
\text { Mean } \pm \text { SD }\end{array}$ & $10.6 \pm 13.5$ & $7.2 \pm 5.6$ & 0.48 \\
\hline
\end{tabular}


in the TRP $(p=0.966)$. The stone burden of any residual kidney stones was reported in Table 2; these are fragments $<1 \mathrm{~cm}$ migrated to the lower pole of the kidney which did not require further treatment. Concomitant pyeloplasty sec. Anderson-Hynes was performed with retroperitoneal approach in 1 (9\%) patient and with transperitoneal approach in $3(33 \%)$ patients, $\mathrm{p}=0.18$.

The drain was on average removed after $4.27 \pm 2.7$ days in the RRP group and after $1 \pm 2.7$ days in the TRP group $(p=0.013)$. The length of hospital stay was lower in the TRP group compared to the RRP group $(2 \pm 2.7$ days in the TRP group vs $5.3 \pm 2.7$ days in the RRP group, $\mathrm{p}=$ 0.013).

\section{Discussion}

The role of robotic assisted surgery continues to develop within the field of urological surgery. In literature, the growing evidence demonstrating that the breadth and complexity of surgical procedures performed using the da Vinci platform is continually expanding $(7,8,4)$. The robotic surgery, whilst maintaining the benefits of standard laparoscopy, provides the surgeon with additional advantages of greater dexterity, a wider range of movement, tremor filtration, three-dimensional vision, and primary surgeon camera control; moreover, we observed significant continuous improvement in terms of bleeding, complications, hospital stay and global quality of life of patients (9-11). In the treatment of urolithiasis laparoscopic and robotic surgery represent valid options in specific cases; in particular the coexistence of UPJO and kidney stones is one of the main indications for the laparoscopic treatment (12). Robotic surgery has some advantages over laparoscopic surgery, such as three-dimensional (3D) view, wristed instruments and stable camera.

Although anatomical and stone characteristics play a crucial role in case selection, RP appears to be the technique most widely used, conceivably as parenchymal bleeding and potential nephron loss are avoided. The majority of the existing literature on robotic-assisted renal surgery (in particular partial and radical nephrectomy) describe transperitoneal approach and only few papers are published on retroperitoneal technique. The papers on the transperitoneal approach emphasize the advantages of robotic surgical system including: 3D visualization, increased degrees of freedom of movement, and enhanced-reconstructive capabilities. Based on our experience with laparoscopic retroperitoneal approach for renal cancer (5), we recognized that the retroperitoneal approach combines the advantages of robotic technology (3D visualization, increased degrees of freedom of movements) with the advantages of retroperitoneal approach which includes advantages of direct access to the renal hilum and reduced lesion risk to abdominal organs, earlier return of bowel function and shorter length of hospital stay. A recent meta-analysis has shown that there is no difference in terms of complications, blood loss, time of ischemia, conversion and positive surgical margins in the treatment of renal masses between the retroperitoneal and transperitoneal approach, but a reduction of the operation time with the retroperitoneal approach has been highlighted (13). The use of rigid laparoscopic instruments in the small space of retroperitoneum cavity has made the retroperitoneal approach less used than the transperitoneal approach (14). In the literature, the cases describing the robotic pyelolithotomy were performed with a transperitoneal approach; Atug et al. (15), described transperitoneal robotic pyelolithotomy in 55 patients with concomitant UPJO: the mean operative time was 275.8 minutes, the mean blood loss was $48.6 \mathrm{ml}$, the mean length of hospital stay was 1.1 days and the stone free rate was $100 \%$. In our series, the mean operative time, blood loss, length of hospital stays and stone free rate with transperitoneal approach were respectively 137 $\pm 31 \mathrm{~min}, 94 \pm 104,2 \pm 2.7$ days and $44 \%$. For our knowledge this is the first study which compares pyelolithotomy with a retroperitoneal and transperitoneal approach for the management of renal stones: the mean operative time, blood loss and length of hospital stay was lower with the transperitoneal approach, but the stone free rate was similar. Swearingen et al. (16) in their series evaluated 27 patients treated with robotic pyelolithotomy and nephrolitotomy with transperitoneal or retroperitoneal access: the mean operative time was $182 \mathrm{~min}$, the mean estimated blood loss was $38 \mathrm{ml}$ and the mean length of stay was 1.7 days. In our series the perioperative outcomes are greater than in the retroperitoneal approach, but the retroperitoneal approach can guarantee some advantages: decreased risk of damage of intraperitoneal structures, direct access to the renal hilum, quicker return of bowel function and earlier mobilization of the patient (17).

However, this is a study that contains some limitations such as reduced number of cases evaluated retrospectively, and the absence of long-term follow-up data. Further prospective studies are necessaries in order to better analyse the differences between the retroperitoneal approach in relation to the transperitoneal.

\section{Conclusions}

Robotic pyelolithotomy with retroperitoneal or transperitoneal approach appears to be a safe and effective minimally invasive treatment for some patients with renal stones, in particular the case of staghorn calculi or urinary tract malformations such as UPJO. The transperitoneal approach may give lower operative time and better results in terms of blood loss and length of hospital stay.

\section{REFERENCES}

1. Türk C, Petfik A, Sarica K, et al. EAU Guidelines on Diagnosis and Conservative Management of Urolithiasis Eur Urol. 2016; 69:468-474.

2. Healy KA, Ogan K. Pathophysiology and management of infectious staghorn calculi. Urol Clin North Am. 2007; 34:363-374.

3. Preminger GM, Assimos DG, Lingeman JE, et al. Chapter 1: AUA guideline on management of staghorn calculi: diagnosis and treatment recommendations. J Urol. 2005; 173:1991-2000.

4. Schiavina R, Zaramella S, Chessa F, et al. Laparoscopic and robotic ureteral stenosis repair: a multi-institutional experience with a long-term follow-up. J Robot Surg. 2016; 10:323-330.

5. Porreca A, D'agostino D, Dente D, et al. Retroperitoneal 
approach for robot-assisted partial nephrectomy: technique and early outcomes. Int Braz J Urol. 2018; 44:63-68.

6. Bove P, Iacovelli V, Sandri M, et al.. Entry techniques in laparoscopic radical and partial nephrectomy: a multicenter international survey of contemporary practices. Minerva Urol Nefrol. 2018; 70:414-421.

7. Porreca A, Chessa F, Romagnoli D, et al.Robot assisted radical cystectomy with totally intracorporeal urinary diversion: initial, single-surgeon's experience after a modified modular training. Minerva Urol Nefrol. 2018; 70:193-201.

8. Porreca A., Salvagio A. Dandrea M, et al. Robotic-Assisted Radical Prostatectomy with the Use of Barbed Sutures. Surg Technol Int. 2017; 30:39-43.

9. Noale M, Maggi S, Artibani W, et al. Pros-IT CNR: an Italian prostate cancer monitoring project. Aging Clin Exp Res. 2017; 29:165-172.

10. Porreca A, Noale M, Artibani W, et al. Disease-specific and general health-related quality of life in newly diagnosed prostate cancer patients: the Pros-IT CNR study. Health Qual Life Outcomes. 2018; 16:122.
11. Gacci M, Noale M, Artibani W, et al. Quality of Life After Prostate Cancer Diagnosis: Data from the Pros-IT CNR. Eur Urol Focus. 2017; 3:321-324.

12. Ramakumar S, Lancini V, Chan DY, et al. Laparoscopic pyeloplasty with concomitant pyelolithotomy. J Urol 2002; 167:1378-80.

13. Xia L, Zhang X, Wang X, et al.Transperitoneal versus retroperitoneal robot-assisted partial nephrectomy: A systematic review and meta-analysis. Int J Surg. 2016; 30:109-15.

14. Ng CS, Gill IS, Ramani AP, et al. Transperitoneal versus retroperitoneal laparoscopic partial nephrectomy: patient selection and perioperative outcomes. J Urol. 2005; 174:846-9.

15. Atug F, Castle EP, Burgess SV, et al. Concomitant management of renal calculi and pelvi-ureteric junction obstruction with robotic laparoscopic surgery. BJU Int. 2005; 96:1365-1368.

16. Swearingen $R$, Sood $A$, Madi $R$, et al. Zero-fragment Nephrolithotomy: A Multi-center Evaluation of Robotic Pyelolithotomy and Nephrolithotomy for Treating Renal Stones. Eur Urol. 2017; 72:1014-1021.

17. Hu JC, Treat E, Filson CP, et al. Technique and outcomes of robot-assisted retroperitoneoscopic partial nephrectomy: a multicenter study. Eur Urol. 2014; 66:542-9.

\section{Correspondence}

Daniele D'Agostino, MD (Corresponding Author)

dott.dagostino@gmail.com

Paolo Corsi, MD

pcorsi@casacura.it

Marco Giampaoli, MD

mgiampaoli@casacura.it

Daniele Romagnoli, MD

dromagnoli@casacura.it

Walter Artibani, MD

prof.artibani@gmail.com

Angelo Porreca, MD

angeloporreca@gmail.com

Department of Robotic Urological Surgery, Abano Terme Hospital

Piazza Cristoforo Colombo 1, 35031 Abano Terme (PD) (Italy)

Federico Mineo Bianchi, MD

federico.mineobianchi@gmail.com

Giacomo Saraceni, MD

giacomo.saraceni@gmail.com

Marco Garofalo, MD

marco.garofalo@unibo.it

Riccardo Schiavina, MD

rschiavina@yahoo.it

Eugenio Brunocilla, MD

eugenio.brunocilla@unibo.it

Department of Urology, University of Bologna, Bologna (Italy)

Simone Crivellaro, MD

crivellaro76@hotmail.com

Division of Urology, Department of Surgery, University of Illinois at Chicago,

Chicago, IL, (USA) 